\title{
Robust exponential stability results for uncertain infinite delay differential systems with random impulsive moments
}

\author{
A Vinodkumar ${ }^{1}$, T Senthilkumar ${ }^{1}$ and Xiaodi Li2 ${ }^{2 *}$ (D)
}

"Correspondence: Ixd@sdnu.edu.cn

${ }^{2} S c h o o l$ of Mathematics and Statistics, Shandong Normal University, Ji'nan, P.R. China

Full list of author information is

available at the end of the article

\begin{abstract}
In this paper, we establish some criteria on robust exponential stability by using the formula for the variation of parameters and estimating the Cauchy matrix. More importantly, the robust stability criteria do not require the stability of the corresponding continuous system, and so they can be more widely applied to stabilize the unstable continuous system with time delays and uncertainties by using random impulsive control. Further, we give some numerical examples to illustrate the theoretical results.
\end{abstract}

Keywords: random impulses; exponential stability; robustness; infinite delay differential systems

\section{Introduction}

Uncertainties happen frequently in various engineering, biological, economical, etc. systems. Delay differential systems usually encounter the uncertainties because of system parameters, modeling error or some factors. The uncertainties that affect a time delay system fall into two different categories. They can be classified into delay dependent and delay independent criteria. Since the delay dependent criteria make use of information on the length of delays, they are less conservative than the delay independent ones. It is well known that uncertainties often result in instability. Therefore, the robust stability is performed for generally bounded and uncertain domains. The robust stability has received considerable attention in recent years. Yang and $\mathrm{Xu}$ in [1] presented robust stability for an uncertain impulsive control system with time-varying delay. Li in [2] established robust exponential stability for impulsive systems with state-dependent delays, and several interesting results were established in [3-11].

Impulsive differential systems and impulsive control systems have attracted increasing interest in recent years. Such systems arise in many fields of science and engineering, see $[2,12-20]$. When impulse time is random, the solutions of the differential system behave as a stochastic process. There are several research works in the literature on random impulsive differential systems. Wu et al. in [21] studied the existence and uniqueness of solutions to random impulsive differential systems. In [22] Anguraj and Vinodkumar proved the existence, uniqueness and stability results of random impulsive semilinear differen- 
tial systems. Ravi Agarwal et al.[23] proved exponential stability for differential equations with random impulses at random times. For further study, refer to [21, 22, 24-30] and the references therein. So far there has been no paper reported dealing with uncertain random impulsive delay differential systems. Therefore, it is necessary to investigate the stability of uncertain random impulsive delay differential systems.

The paper is organized as follows. In Section 2, we recall briefly the notations, definitions, lemmas and preliminaries which are used throughout this paper. In Section 3, we prove robust exponential stability of uncertain random impulsive linear and nonlinear infinite delay differential systems by using the method of variation of parameters. Finally, in Section 4, we give some examples to illustrate our result.

\section{Preliminaries}

Let $\Re^{n}$ be the $n$-dimensional Euclidean space and $\Omega$ be a nonempty set. Assume that $\left\{\tau_{k}\right\}_{k=1}^{\infty}$ is a sequence of independent exponentially distributed random variables with parameter $\lambda$, and each random variable $\tau_{k}$ is defined from $\Omega$ to $D_{k} \stackrel{\text { def. }}{=}\left(0, d_{k}\right)$ for $k=1,2, \ldots$, where $0<d_{k}<+\infty$. Let us denote by $\left\{B_{t}, t \geq 0\right\}$ the simple counting process generated by $\left\{\xi_{n}\right\}$, that is, $\left\{B_{t} \geq n\right\}=\left\{\xi_{n} \leq t\right\}$, and denote by $\mathcal{F}_{t}$ the $\sigma$-algebra generated by $\left\{B_{t}, t \geq 0\right\}$. Then $\left(\Omega, P,\left\{\mathcal{F}_{t}\right\}\right)$ is a probability space. $E$ stands for the mathematical expectation operator with respect to the given probability measure $P$.

For $x \in \Re^{n}$ and $A \in \Re^{n \times n}$, the norm is defined as follows:

$$
\|x\|=\sqrt{\sum_{j=1}^{n} x_{j}^{2}}, \quad\|A\|=\sqrt{\lambda_{\max }\left(A^{T} A\right)}, \quad \mu(A)=\frac{1}{2} \lambda_{\max }\left(A+A^{T}\right),
$$

where $\lambda_{\max }(\cdot)$ is the largest eigenvalue of the matrix.

Consider the following nonlinear uncertain random impulsive control system with infinite delays:

$$
\begin{aligned}
\dot{x}(t)= & (A+\Delta A) x(t)+(B+\Delta B) x^{\mu_{1}}(t-\tau(t)) \\
& +(C+\Delta C) \int_{0}^{\infty} h(\eta) x^{\mu_{2}}(t-\eta) d \eta, \quad t \neq \xi_{k}, t \geq t_{0}, \\
x\left(\xi_{k}^{+}\right)= & b_{i}\left(\tau_{i}\right) x\left(\xi_{k}^{-}\right), \quad k=1,2, \ldots, \\
x(s)= & \varphi(s), \quad s \leq t_{0},
\end{aligned}
$$

where $\mu_{1} \geq 1, \mu_{2} \geq 1$. Let $\mathcal{P C}\left(\left(-\infty, t_{0}\right], \Re^{n}\right)=\left\{\varphi:\left(-\infty, t_{0}\right] \rightarrow \Re^{n}, \varphi(t)\right.$ is piecewise continuous $\}$, and for $\varphi \in \mathcal{P C}\left(\left(-\infty, t_{0}\right], \Re^{n}\right)$, the norm is defined as $E\|\varphi\|^{2}=$ $\sup _{t \leq t_{0}} E\|\varphi(t)\|^{2} ; A, B, C \in \Re^{n \times n}$ are matrices; $\tau(t)$ is the time-varying delay function with $0 \leq \tau(t) \leq \tau, \tau$ is a given positive constant; $\Delta A, \Delta B, \Delta C$ are the uncertain matrices, which vary within the range of $\|\Delta A\| \leq a,\|\Delta B\| \leq b,\|\Delta C\| \leq c$, where $a, b, c$ are known nonnegative constants; $h(s) \in C\left(\Re^{+}, \Re\right)$ satisfies $\int_{0}^{+\infty}|h(s)| e^{\mu_{2} \eta s} d s<\infty$, where $\eta>0$ is a given constant; $b_{k}: D_{k} \rightarrow \Re^{n \times n}$ is a matrix-valued function for each $k=1,2, \ldots ; \xi_{0}=t_{0}$ and $\xi_{k}=\xi_{k-1}+\tau_{k}$ for $k=1,2, \ldots$, here $t_{0} \in \Re$ is an arbitrary real number. Obviously, $t_{0}=\xi_{0}<\xi_{1}<\xi_{2}<\cdots<\lim _{k \rightarrow \infty} \xi_{k}=\infty ; x\left(\xi_{k}^{-}\right)=\lim _{t \uparrow \xi_{k}} x(t)$ according to their paths with the norm $E\|x\|^{2}=\sup _{t_{0} \leq s \leq t} E\|x(s)\|^{2}$ for each $t$ satisfying $t \geq t_{0}$. 
When $\mu_{1}, \mu_{2}=1$, system (1) becomes the linear uncertain random impulsive control system with infinite delays of the form

$$
\begin{aligned}
& \dot{x}(t)=(A+\Delta A) x(t)+(B+\Delta B) x(t-\tau(t)) \\
&+(C+\Delta C) \int_{0}^{+\infty} h(\eta) x(t-\eta) d \eta, \quad t \neq \xi_{k}, t \geq t_{0}, \\
& x\left(\xi_{k}^{+}\right)=b_{i}\left(\tau_{i}\right) x\left(\xi_{k}^{-}\right), \quad k=1,2, \ldots, \\
& x(s)=\varphi(s), \quad s \leq t_{0} .
\end{aligned}
$$

If $h(\eta)=0$, then system (2) becomes the linear uncertain random impulsive control system with infinite delays.

$$
\begin{aligned}
& \dot{x}(t)=(A+\Delta A) x(t)+(B+\Delta B) x(t-\tau(t)), \quad t \neq \xi_{k}, t \geq t_{0}, \\
& x\left(\xi_{k}^{+}\right)=b_{i}\left(\tau_{i}\right) x\left(\xi_{k}^{-}\right), \quad k=1,2, \ldots, \\
& x(s)=\varphi(s), \quad s \leq t_{0} .
\end{aligned}
$$

In particular, $\Delta A, \Delta B=0$ then system (3) becomes the random impulsive control system with infinite delays.

$$
\begin{aligned}
& \dot{x}(t)=A x(t)+B x(t-\tau(t)), \quad t \neq \xi_{k}, t \geq t_{0}, \\
& x\left(\xi_{k}^{+}\right)=b_{i}\left(\tau_{i}\right) x\left(\xi_{k}^{-}\right), \quad k=1,2, \ldots, \\
& x(s)=\varphi(s), \quad s \leq t_{0} .
\end{aligned}
$$

We always assume that the solution $x(t)$ of (1) is continuous on the right and limitable on the left. Now, we introduce the following lemma and hypotheses used in our discussion.

Lemma 1 ([23]) The probability that there will be exactly $k$ impulses until the time $t, t \geq t_{0}$, where impulse moments $\xi_{k}, k=1,2, \ldots$, follow exponential distribution with parameter $\lambda$, is given by the equality $P\left(I_{\left[\xi_{k}, \xi_{k+1}\right)}(t)\right)=\frac{\lambda^{k}\left(t-t_{0}\right)^{k}}{k !} e^{-\lambda\left(t-t_{0}\right)}$, where the events $I_{\left[\xi_{k}, \xi_{k+1}\right)}(t)=\{\omega \in$ $\left.\Omega: \xi_{k}(\omega)<t<\xi_{k+1}(\omega)\right\}, k=1,2, \ldots$

Remark 1 From [23], the expected value of solution $x(t)$ for the random impulsive differential equations is given as

$$
E[\|x(t)\|]=\sum_{k=0}^{\infty} E\left[\|x(t)\| \mid I_{\left[\xi_{k}, \xi_{k+1}\right)}(t)\right] P\left(I_{\left[\xi_{k}, \xi_{k+1}\right)}(t)\right),
$$

where the impulse moments $\xi_{k}, k=1,2, \ldots$, follow exponential distribution with parameter $\lambda$.

Definition 1 Assume $x(t)=x\left(t, t_{0}, \varphi\right)$ to be the solution of (1) through $\left(t_{0}, \varphi\right)$. Then the zero solution of (1) is said to be globally exponentially mean square stable if, for any initial data $x_{t_{0}}=\varphi$, there exist two positive numbers $\gamma>0, M \geq 1$ such that

$$
E\|x(t)\|^{2} \leq M E\|\varphi\|^{2} e^{-\gamma\left(t-t_{0}\right)}, \quad t \geq t_{0} .
$$


Remark 2 The uncertain random impulsive dynamical system (1) is called robust exponentially mean square stable if the zero solution $x=0$ of the system is globally exponentially mean square stable for any $\|\Delta A\| \leq a,\|\Delta B\| \leq b,\|\Delta C\| \leq c$, where $a, b, c$ are known nonnegative constants.

Hypothesis $\left(\mathbf{H}_{1}\right)$ The condition $E\left\{\max _{i, k}\left\{\prod_{j=i}^{k}\left\|b_{j}\left(\tau_{j}\right)\right\|\right\}\right\}$ is uniformly bounded. That is, there is a constant $\alpha>0$ such that

$$
E\left\{\max _{i, k}\left\{\prod_{j=i}^{k}\left\|b_{j}\left(\tau_{j}\right)\right\|\right\}\right\} \leq \alpha \quad \text { for all } \tau_{j} \in D_{j}, j=1,2, \ldots
$$

\section{Main results}

We need the following lemma to prove the main results.

Lemma 2 Let $\phi\left(t, t_{0}\right)$ be the Cauchy matrix of the linear system:

$$
\begin{aligned}
& \dot{x}(t)=A x(t), \quad t \neq \xi_{k}, t \geq t_{0} \\
& x\left(\xi_{k}^{+}\right)=b_{k}\left(\tau_{k}\right) x\left(\xi_{k}^{-}\right), \\
& x(s)=\varphi(s), \quad s \leq t_{0} .
\end{aligned}
$$

Then it satisfies $\left\|\phi\left(t, t_{0}\right)\right\| \leq e^{[\mu(A)-\lambda(1-\alpha)]\left(t-t_{0}\right)}, t \geq t_{0}$.

Proof For any $x_{0} \in \Re^{n}$, let $x(t)=x\left(t, t_{0}, x_{0}\right)$ be a solution through $\left(t_{0}, x_{0}\right)$. Calculating the upper right derivative $D^{+}\|x(t)\|$ along the solution $x(t)$ of equation (5), we have

$$
D^{+}\|x(t)\| \leq \mu(A)\|x(t)\|, \quad t \neq \xi_{k}, t \geq t_{0}
$$

and

$$
\begin{aligned}
\|x(t)\|^{2} \leq & \left.\leq\left\|\prod_{j=1}^{k} b_{j}\left(\tau_{j}\right)\right\| e^{\mu(A)\left(t-t_{0}\right)}\left\|x\left(t_{0}\right)\right\| I_{\left[\xi_{k}, \xi_{k+1}\right]}(t)\right]^{2}, \quad t \in\left[\xi_{k}, \xi_{k+1}\right], t \geq t_{0}, \\
E\|x(t)\|^{2} & \leq \sum_{k=0}^{\infty} \prod_{j=1}^{k}\left\|b_{j}\left(\tau_{j}\right)\right\|^{2} e^{\mu(A)\left(t-t_{0}\right)} E\left\|x\left(t_{0}\right)\right\|^{2} P\left(I_{\left[\xi_{k}, \xi_{k+1}\right]}(t)\right), \quad t \geq t_{0}, \\
E\|x(t)\|^{2} & \leq e^{\mu(A)\left(t-t_{0}\right)} E\left\|x\left(t_{0}\right)\right\|^{2} \sum_{i=0}^{\infty} \frac{\alpha \lambda^{i}\left(t-t_{0}\right)^{i}}{i !} e^{-\lambda\left(t-t_{0}\right)}, \quad t \geq t_{0} \\
& \leq e^{\mu(A)\left(t-t_{0}\right)} E\left\|x\left(t_{0}\right)\right\|^{2} e^{-(1-\alpha) \lambda\left(t-t_{0}\right)}, \\
E\|x(t)\|^{2} & \leq e^{[\mu(A)-\lambda(1-\alpha)]\left(t-t_{0}\right)} E\left\|x\left(t_{0}\right)\right\|^{2} .
\end{aligned}
$$

Since $x(t)=\phi\left(t, t_{0}\right) x\left(t_{0}\right)$, we obtain

$$
\begin{aligned}
\left\|\phi\left(t, t_{0}\right)\right\| & =\sup _{\left\|x\left(t_{0}\right)\right\| \neq 0} \frac{e^{[\mu(A)-\lambda(1-\alpha)]\left(t-t_{0}\right)} E\left\|x\left(t_{0}\right)\right\|^{2}}{E\left\|x\left(t_{0}\right)\right\|^{2}}, \\
\left\|\phi\left(t, t_{0}\right)\right\| & \leq e^{[\mu(A)-\lambda(1-\alpha)]\left(t-t_{0}\right)}, \quad t \geq t_{0} .
\end{aligned}
$$

This completes the proof. 
Now consider the linear uncertain random impulsive control system (3).

Theorem 1 Assume that hypothesis $\left(\mathrm{H}_{1}\right)$ holds, then the zero solution of system (3) is robustly exponentially stable provided $2 a^{2}+2(b+\|B\|)^{2}+2 k<0$, where $k=[\mu(A)-\lambda(1-\alpha)]$.

Proof Since $2 a^{2}+2(b+\|B\|)^{2}+2 k<0$, we choose small enough $\gamma \in(0, \eta)$ such that $2 a^{2}+$ $2(b+\|B\|)^{2} e^{\gamma\left(\tau-t_{0}\right)}+2 k+\gamma<0$, and $e^{-\gamma t_{0}} \leq 1$.

Furthermore, for any $\epsilon \in(0, \gamma)$, we have

$$
0 \leq 2 a^{2}+2(b+\|B\|)^{2} e^{(\gamma-\epsilon)\left(\tau-t_{0}\right)} \leq-(2 k+\gamma-\epsilon) .
$$

By the formula for variation of parameters, the solution of (3) can be presented as follows:

$$
x(t)=\phi\left(t, t_{0}\right) x\left(t_{0}\right)+\int_{t_{0}}^{t} \phi(t, s)[\Delta A x(s)+(B+\Delta B) x(s-r(s))] d s
$$

where $\phi\left(t, t_{0}\right)$ is the Cauchy matrix of the impulsive linear system (5). Then we have

$$
\begin{aligned}
\|x(t)\|^{2} \leq & 2\left\|\phi\left(t, t_{0}\right)\right\|^{2}\left\|x\left(t_{0}\right)\right\|^{2} \\
& +2\left[\int_{t_{0}}^{t}\|\phi(t, s)\|(a\|x(s)\|+(b+\|B\|)\|x(s-r(s))\|) d s\right]^{2}, \\
E\|x(t)\|^{2} \leq & 2\left\|\phi\left(t, t_{0}\right)\right\|^{2} E\left\|x\left(t_{0}\right)\right\|^{2} \\
& +2\left[\int_{t_{0}}^{t}\|\phi(t, s)\|(a E\|x(s)\|+(b+\|B\|) E\|x(s-r(s))\|) d s\right]^{2}, \\
E\|x(t)\|^{2} \leq & 2 e^{2 k\left(t-t_{0}\right)} E\left\|x\left(t_{0}\right)\right\|^{2} \\
& +2 \int_{t_{0}}^{t} e^{2 k(t-s)}\left(2 a^{2} E\|x(s)\|^{2}+2(b+\|B\|)^{2} E\|x(s-r(s))\|^{2}\right) d s, \quad t \geq t_{0} .
\end{aligned}
$$

Without loss of generality, we assume that $E\|\varphi\|^{2}>0$. From $\gamma>\epsilon$, we get

$$
E\|x(t)\|^{2} \leq E\|\varphi\|^{2}<E\|\varphi\|^{2} e^{-(\gamma-\epsilon)\left(t-t_{0}\right)} \quad \text { for } t \leq t_{0} .
$$

In the following, we shall prove that

$$
E\|x(t)\|^{2}<E\|\varphi\|^{2} e^{-(\gamma-\epsilon)\left(t-t_{0}\right)} \quad \text { for } t \geq t_{0} .
$$

If this is not true, by (8) and the piecewise continuity of $x(t)$, there must exist $t^{*}>t_{0}$ such that

$$
\begin{aligned}
& E\left\|x\left(t^{*}\right)\right\|^{2} \geq E\|\varphi\|^{2} e^{-(\gamma-\epsilon)\left(t^{*}-t_{0}\right)}, \\
& E\|x(t)\|^{2} \leq E\|\varphi\|^{2} e^{-(\gamma-\epsilon)\left(t-t_{0}\right)}, \quad t<t^{*} .
\end{aligned}
$$


From (6), (7) and (11), we get

$$
\begin{aligned}
E\left\|x\left(t^{*}\right)\right\|^{2} \leq & 2 e^{2 k\left(t^{*}-t_{0}\right)} E\|\varphi\|^{2} \\
& +2 \int_{t_{0}}^{t^{*}} e^{2 k\left(t^{*}-s\right)}\left(2 a^{2} e^{-(\gamma-\epsilon) s} E\|\varphi\|^{2}+2(b+\|B\|)^{2} e^{-(\gamma-\epsilon)(s-r(s))} E\|\varphi\|^{2}\right) d s \\
\leq & 2 e^{2 k\left(t^{*}-t_{0}\right)} E\|\varphi\|^{2} \\
& +2 \int_{t_{0}}^{t^{*}} e^{2 k\left(t^{*}-s\right)} e^{-(\gamma-\epsilon) s} E\|\varphi\|^{2}\left(2 a^{2}+2(b+\|B\|)^{2} e^{(\gamma-\epsilon)(r(s))}\right) d s \\
\leq & 2 e^{2 k\left(t^{*}-t_{0}\right)} E\|\varphi\|^{2} \\
& +2 e^{2 k t^{*}} E\|\varphi\|^{2}\left(2 a^{2}+2(b+\|B\|)^{2} e^{(\gamma-\epsilon)(r(s))}\right) \int_{t_{0}}^{t^{*}} e^{-(2 k+(\gamma-\epsilon)) s} d s \\
\leq & 2 e^{2 k\left(t^{*}-t_{0}\right)} E\|\varphi\|^{2} \\
& \times\left[1+\left(2 a^{2}+2(b+\|B\|)^{2} e^{(\gamma-\epsilon)\left(\tau-t_{0}\right)}\right) e^{2 k t_{0}} \int_{t_{0}}^{t^{*}} e^{-(2 k+\gamma-\epsilon) s} d s\right] \\
\leq & 2 e^{2 k\left(t^{*}-t_{0}\right)} E\|\varphi\|^{2}\left(1+\left(2 a^{2}+2(b+\|B\|)^{2} e^{(\gamma-\epsilon)\left(\tau-t_{0}\right)}\right) \times(-(2 k+\gamma-\epsilon))^{-1}\right. \\
& \left.\times\left\{e^{2 k t_{0}}\left[e^{-(2 k+\gamma-\epsilon) t^{*}}-e^{-(2 k+\gamma-\epsilon) t_{0}}\right]\right\}\right) \\
\leq & 2 e^{2 k\left(t^{*}-t_{0}\right)} E\|\varphi\|^{2}\left[1+e^{-2 k\left(t^{*}-t_{0}\right)-(\gamma-\epsilon) t^{*}}-1\right] \\
\leq & 2 e^{2 k\left(t^{*}-t_{0}\right)} E\|\varphi\|^{2}\left(e^{-2 k\left(t^{*}-t_{0}\right)-(\gamma-\epsilon) t^{*}}\right), \\
\leq & 2 E\|\varphi\|^{2} e^{-(\gamma-\epsilon) t^{*}} \cdot \\
E\left(t^{*}\right) \|^{2} & {[1] }
\end{aligned}
$$

This contradicts (10), and so estimate (9) holds. Letting $\epsilon \rightarrow 0$, we have

$$
E\|x(t)\|^{2} \leq 2 E\|\varphi\|^{2} e^{-\gamma t}, \quad t \geq t_{0} .
$$

This completes the proof.

In the following theorem, we prove that the nonlinear uncertain random impulsive control system (1) is robust exponentially mean square stable.

Theorem 2 Assume that hypothesis $\left(\mathrm{H}_{1}\right)$ holds, then the zero solution of system (1) is robustly exponentially stable provided $4 a^{2}+4(b+\|B\|)^{2} E\|\varphi\|^{2\left(\mu_{1}-1\right)}+2(c+\|C\|)^{2} \times$ $E\|\varphi\|^{2\left(\mu_{2}-1\right)} M+2 k<0$, where $k=[\mu(A)-\lambda(1-\alpha)]$ and $M=\int_{0}^{\infty}\|h(s)\| e^{\mu_{2} \eta s} d s$.

Proof Since $4 a^{2}+4(b+\|B\|)^{2} E\|\varphi\|^{2\left(\mu_{1}-1\right)}+2(c+\|C\|)^{2} E\|\varphi\|^{2\left(\mu_{2}-1\right)} M+2 k<0$, we choose small enough $\gamma \in(0, \eta)$ such that

$$
4 a^{2}+4(b+\|B\|)^{2} E\|\varphi\|^{2\left(\mu_{1}-1\right)} e^{\mu_{1} \gamma\left(\tau-t_{0}\right)}+2(c+\|C\|)^{2} E\|\varphi\|^{2\left(\mu_{2}-1\right)} M+(2 k+\gamma)<0,
$$

and

$$
e^{-\gamma t_{0}} \leq 1
$$


Furthermore, for any $\epsilon \in(0, \gamma)$, we have

$$
\begin{aligned}
0 & \leq 4 a^{2}+4(b+\|B\|)^{2} E\|\varphi\|^{2\left(\mu_{1}-1\right)} e^{\mu_{1}(\gamma-\epsilon)\left(\tau-t_{0}\right)}+2(c+\|C\|)^{2} E\|\varphi\|^{2\left(\mu_{2}-1\right)} M \\
& \leq-(2 k+\gamma-\epsilon) .
\end{aligned}
$$

By the formula for variation of parameters, the solution $x(t)$ can be represented as

$$
\begin{aligned}
x(t)= & \phi\left(t, t_{0}\right) x\left(t_{0}\right)+\int_{t_{0}}^{t} \phi(t, s)\left(\Delta A x(s)+(B+\Delta B) x^{\mu_{1}}(s-r(s))\right. \\
& \left.+(C+\Delta C) \int_{0}^{\infty} h(\eta) x^{\mu_{2}}(s-\eta) d \eta\right) d s,
\end{aligned}
$$

where $\phi\left(t, t_{0}\right)$ is the Cauchy matrix of impulsive linear system (5). Then we have

$$
\begin{aligned}
\|x(t)\|^{2} \leq 2 & \left\|\phi\left(t, t_{0}\right)\right\|^{2}\left\|x\left(t_{0}\right)\right\|^{2} \\
& +2\left(\int _ { t _ { 0 } } ^ { t } \| \phi ( t , s ) \| \left(a\|x(s)\|+(b+\|B\|)\left\|x^{\mu_{1}}(s-r(s))\right\|\right.\right. \\
& \left.\left.+(c+\|C\|) \int_{0}^{\infty}\|h(\eta)\|\left\|x^{\mu_{2}}(s-\eta)\right\| d \eta\right) d s\right)^{2}, \\
E\|x(t)\|^{2} \leq & 2 e^{2 k\left(t-t_{0}\right)} E\left\|x\left(t_{0}\right)\right\|^{2} \\
& +2\left(\int _ { t _ { 0 } } ^ { t } e ^ { k ( t - s ) } \left(a E\|x(s)\|+(b+\|B\|) E\left\|x^{\mu_{1}}(s-r(s))\right\|\right.\right. \\
& \left.\left.+(c+\|C\|) \int_{0}^{\infty}\|h(\eta)\| E\left\|x^{\mu_{2}}(s-\eta)\right\| d \eta\right) d s\right)^{2} \\
\leq & 2 e^{2 k\left(t-t_{0}\right)} E\left\|x\left(t_{0}\right)\right\|^{2} \\
& +2 \int_{t_{0}}^{t} e^{2 k(t-s)} d s\left(a E\|x(s)\|+(b+\|B\|) E\left\|x^{\mu_{1}}(s-r(s))\right\|\right. \\
& \left.+(c+\|C\|) \int_{0}^{\infty}\|h(\eta)\| E\left\|x^{\mu_{2}}(s-\eta)\right\| d \eta\right)^{2}, \\
E\|x(t)\|^{2} \leq & 2 e^{2 k\left(t-t_{0}\right)} E\left\|x\left(t_{0}\right)\right\|^{2} \\
& +2\left(4 a^{2} E\|x(s)\|^{2}+4(b+\|B\|)^{2} E\left\|x^{\mu_{1}}(s-r(s))\right\|^{2}\right. \\
& \left.+2(c+\|C\|)^{2} \int_{0}^{\infty}\|h(\eta)\| E\left\|x^{\mu_{2}}(s-\eta)\right\|^{2} d \eta\right) \int_{t_{0}}^{t} e^{2 k(t-s)} d s .
\end{aligned}
$$

Without loss of generality, we assume that $E\|\varphi\|^{2}>0$. From $\gamma>\epsilon$, it is easily observed that

$$
E\|x(t)\|^{2} \leq E\|\varphi\|^{2}<E\|\varphi\|^{2} e^{-(\gamma-\epsilon)\left(t-t_{0}\right)} \quad \text { for } t \leq t_{0} .
$$

We shall prove that

$$
E\|x(t)\|^{2} \leq E\|\varphi\|^{2}<E\|\varphi\|^{2} e^{-(\gamma-\epsilon)\left(t-t_{0}\right)} \quad \text { for } t \geq t_{0} .
$$


If this is not true, by (14) and the piecewise continuity of $x(t)$, there must exist $t^{*}>t_{0}$ such that

$$
\begin{aligned}
& E\left\|x\left(t^{*}\right)\right\|^{2} \geq E\|\varphi\|^{2} e^{-(\gamma-\epsilon)\left(t^{*}-t_{0}\right)}, \\
& E\|x(t)\|^{2} \leq E\|\varphi\|^{2} e^{-(\gamma-\epsilon)\left(t-t_{0}\right)}, \quad t<t^{*} .
\end{aligned}
$$

From (12), (13) and (17), we get

$$
\begin{aligned}
E\left\|x\left(t^{*}\right)\right\|^{2} \leq & 2 e^{2 k\left(t^{*}-t_{0}\right)} E\|\varphi\|^{2}+2 \int_{t_{0}}^{t^{*}} e^{2 k\left(t^{*}-s\right)} \\
& \times\left(4 a^{2} e^{-(\gamma-\epsilon) s} E\|\varphi\|^{2}+4(b+\|B\|)^{2} e^{-\mu_{1}(\gamma-\epsilon)(s-r(s))} E\|\varphi\|^{2 \mu_{1}}\right. \\
& \left.+2(c+\|C\|)^{2} \int_{0}^{\infty}\|h(\eta)\| e^{-\mu_{2}(\gamma-\epsilon)(s-\eta)} E\|\varphi\|^{2 \mu_{1}} d \eta\right) d s \\
\leq & 2 e^{2 k\left(t^{*}-t_{0}\right)} E\|\varphi\|^{2} \\
& +2 e^{2 k t^{*}} E\|\varphi\|^{2} \int_{t_{0}}^{t^{*}} e^{-(2 k+\gamma-\epsilon) s} \\
& \times\left(4 a^{2}+4(b+\|B\|)^{2} E\|\varphi\|^{2\left(\mu_{1}-1\right)} e^{(\gamma-\epsilon)\left(s-\mu_{1} s+\mu_{1} r(s)\right)}\right. \\
& \left.+2(c+\|C\|)^{2} E\|\varphi\|^{2\left(\mu_{2}-1\right)} \int_{0}^{\infty}\|h(\eta)\| e^{(\gamma-\epsilon)\left(s-\mu_{2} s+\mu_{2} \eta\right)} d \eta\right) d s \\
\leq & 2 e^{2 k\left(t^{*}-t_{0}\right)} E\|\varphi\|^{2} \\
& +2 e^{2 k t^{*}} E\|\varphi\|^{2}\left(4 a^{2}+4(b+\|B\|)^{2} E\|\varphi\|^{2\left(\mu_{1}-1\right)} e^{\mu_{1}(\gamma-\epsilon)\left(\tau-t_{0}\right)}\right. \\
& \left.+2(c+\|C\|)^{2} E\|\varphi\|^{2\left(\mu_{2}-1\right)} M\right) \int_{t_{0}}^{t^{*}} e^{-(2 k+\gamma-\epsilon) s} d s \\
\leq & 2 e^{2 k\left(t^{*}-t_{0}\right)} E\|\varphi\|^{2} \\
& +2 e^{2 k t^{*}} E\|\varphi\|^{2}\left(4 a^{2}+4(b+\|B\|)^{2} E\|\varphi\|^{2\left(\mu_{1}-1\right)} e^{\mu_{1}(\gamma-\epsilon)\left(\tau-t_{0}\right)}\right. \\
& +2(c+\|C\|)^{2} E\|\varphi\|^{2\left(\mu_{2}-1\right)} M \\
& \left.\times(-(2 k+\gamma-\epsilon))^{-1}\right)\left[e^{-(2 k+\gamma-\epsilon) t^{*}}-e^{-(2 k+\gamma-\epsilon) t_{0}}\right] \\
\leq & 2 e^{2 k\left(t^{*}-t_{0}\right)} E\|\varphi\|^{2}\left[1+e^{2 k t_{0}}\left(e^{-(2 k+\gamma-\epsilon) t^{*}}-e^{\left.-(2 k+\gamma-\epsilon) t_{0}\right)}\right]\right. \\
\leq & 2 e^{2 k\left(t^{*}-t_{0}\right)} E\|\varphi\|^{2}\left[1+e^{-2 k\left(t^{*}-t_{0}\right)-(\gamma-\epsilon) t^{*}}-1\right], \\
\leq & 2 E\|\varphi\|^{2} e^{-(\gamma-\epsilon) t^{*}} . \\
E x\left(t^{*}\right) \|^{2} & \\
& \\
&
\end{aligned}
$$

This contradicts (16), and so estimate (15) holds. Letting $\epsilon \rightarrow 0$, we have

$$
E\|x(t)\|^{2} \leq 2 E\|\varphi\|^{2} e^{-\gamma t}, \quad t \geq t_{0} .
$$

This completes the proof.

Especially, for the linear case, we have the following result. 
Corollary 1 Assume that hypothesis $\left(\mathrm{H}_{1}\right)$ holds, then the zero solution of system (2) is robustly exponentially stable provided $4 a^{2}+4(b+\|B\|)^{2}+2(c+\|C\|)^{2} M+2 k<0$, where $k=[\mu(A)-\lambda(1-\alpha)]$ and $M=\int_{0}^{\infty}\|h(\eta)\| e^{(\gamma-\epsilon) \eta} d \eta$.

Proof The proof is similar to that of Theorem 2, when $\mu_{1}=1, \mu_{2}=1$.

\section{Example}

In this section, we will give four numerical examples to illustrate that our results can be applied to stabilize the unstable continuous systems by using random impulsive control.

Example 1 Consider the following linear uncertain random impulsive control system with infinite delays:

$$
\begin{aligned}
& \dot{x}(t)=(A+\Delta A) x(t)+(B+\Delta B) x(t-\tau(t)) \\
&+(C+\Delta C) \int_{0}^{\infty} h(\eta) x(t-\eta) d \eta, \quad t \neq \xi_{k}, t \geq t_{0}, \\
& x\left(\xi_{k}^{+}\right)=b_{i}\left(\tau_{i}\right) x\left(\xi_{k}^{-}\right), \quad k=1,2, \ldots,
\end{aligned}
$$

where $\tau(t)$ is a time-varying delay function with $\tau(t) \in[0, \tau], h(\eta)=0.1 e^{-1.2 \eta}, \eta>0$ and with the following parameter matrices:

$$
\begin{aligned}
& A=\left[\begin{array}{cc}
1.2 & -1.1 \\
0.7 & 0.8
\end{array}\right], \\
& B=\left[\begin{array}{ll}
-1.3 & 0.7 \\
-0.9 & 0.5
\end{array}\right], \\
& C=\left[\begin{array}{cc}
-0.1 & -0.22 \\
0.43 & 0.65
\end{array}\right] .
\end{aligned}
$$

The zero solution of system (18) is robust exponentially mean square stable provided $\lambda(1-$ $\alpha)>9.4263$.

Proof By Corollary 1 , and let us take $\eta=0.2$, then $M=0.1 \int_{0}^{\infty} e^{-\eta} d \eta=0.1$. Now, the eigenvalues of $A$ are $1+0.8544 i, 1-0.8544 i$. Further, we use the defined matrix norm and the matrix measure to get $\mu(A)=1.2828,\|B\|=1.7999,\|C\|=0.8151$ and $\|\Delta A\| \leq a=0.1$, $\|\Delta B\| \leq b=0.2,\|\Delta C\| \leq c=0.3$.

$$
\begin{aligned}
& 4 a^{2}+4(b+\|B\|)^{2}+2(c+\|C\|)^{2} M+2 k<0, \\
& 4 a^{2}+4(b+\|B\|)^{2}+2(c+\|C\|)^{2} M+2(\mu(A)-\lambda(1-\alpha))<0, \\
& 4(0.1)^{2}+4(0.2+1.7999)^{2}+2(0.3+0.8151)^{2}(0.1)+2(1.2828-\lambda(1-\alpha))<0, \\
& 18.8526-2 \lambda(1-\alpha)<0, \\
& \lambda(1-\alpha)>9.4263 .
\end{aligned}
$$

Hence (18) is robust exponentially mean square stable. 
Example 2 Consider the following nonlinear uncertain random impulsive infinite delay differential system:

$$
\begin{aligned}
\dot{x}(t)= & (A+\Delta A) x(t)+(B+\Delta B) x^{\mu_{1}}(t-\tau(t)) \\
& +(C+\Delta C) \int_{0}^{\infty} h(\eta) x^{\mu_{2}}(t-\eta) d \eta, \quad t \neq \xi_{k}, t \geq t_{0}, \\
x\left(\xi_{k}^{+}\right)= & b_{i}\left(\tau_{i}\right) x\left(\xi_{k}^{-}\right), \quad k=1,2, \ldots,
\end{aligned}
$$

where $\mu_{1}, \mu_{2} \geq 1$ with $A, B$ and $C$ defined as in Example 1 . Then there exists a constant $\eta>$ 0 such that $M=\int_{0}^{\infty}|h(s)| e^{\mu_{2} \eta s} d s<\infty$. Then the zero solution of system (19) is robust exponentially mean square stable provided $2.6056+15.9984 E\|\varphi\|^{2\left(\mu_{1}-1\right)}+2.4868 M E\|\varphi\|^{2\left(\mu_{2}-1\right)}-$ $2 \lambda(1-\alpha)<0$.

Proof By Theorem 2, we get

$$
\begin{aligned}
& 4 a^{2}+4(b+\|B\|)^{2} E\|\varphi\|^{2\left(\mu_{1}-1\right)}+2(c+\|C\|)^{2} E\|\varphi\|^{2\left(\mu_{2}-1\right)} M+2 k<0, \\
& 2.6056+15.9984 E\|\varphi\|^{2\left(\mu_{1}-1\right)}+2.4868 M E\|\varphi\|^{2\left(\mu_{2}-1\right)}-2 \lambda(1-\alpha)<0 .
\end{aligned}
$$

Hence (19) is robust exponentially mean square stable.

Example 3 Consider the following uncertain random impulsive control system with infinite delays:

$$
\begin{aligned}
& \dot{x}(t)=(A+\Delta A) x(t)+(B+\Delta B) x(t-\tau(t)), \quad t \neq \xi_{k}, t \geq t_{0}, \\
& x\left(\xi_{k}^{+}\right)=b_{i}\left(\tau_{i}\right) x\left(\xi_{k}^{-}\right), \quad k=1,2, \ldots
\end{aligned}
$$

We take the following parameter matrices:

$$
\begin{aligned}
A & =\left[\begin{array}{ccc}
-1 & 0 & 0.5 \\
0.5 & 2.5 & -1.5 \\
0 & 3 & -1.5
\end{array}\right], \\
B & =\left[\begin{array}{ccc}
-0.5 & 0.1 & 0.3 \\
0.2 & -0.5 & 0.1 \\
-0.3 & 0 & 0.2
\end{array}\right] .
\end{aligned}
$$

Then the zero solution of system (20) is robust exponentially mean square stable provided $\lambda(1-\alpha)>6.4562$.

Proof Checking the eigenvalues of $A$, we find that they are $-0.5,0,0.5$. By Theorem 1 , the matrix norm and the matrix measure are defined as $\mu(A)=2.6591,\|B\|=0.7353$, and $\|\Delta A\| \leq a=1.2,\|\Delta B\| \leq b=0.8$. Then

$$
\begin{aligned}
& 2 a^{2}+2(b+\|B\|)^{2}+2 k<0, \\
& 2 a^{2}+2(b+\|B\|)^{2}+2(\mu(A)-\lambda(1-\alpha))<0,
\end{aligned}
$$




$$
\begin{aligned}
& 2(1.2)^{2}+2(2.3571)+2(2.6591-\lambda(1-\alpha))<0, \\
& 12.9124-2 \lambda(1-\alpha)<0, \\
& \lambda(1-\alpha)>6.4562 .
\end{aligned}
$$

Hence (20) is robust exponentially mean square stable.

Example 4 Consider the following linear random impulsive delay differential system of the form (4):

$$
\left[\begin{array}{l}
x_{1}(t) \\
x_{2}(t)
\end{array}\right]^{\prime}=\left[\begin{array}{cc}
0 & 1 \\
-0.012 & 1
\end{array}\right]\left[\begin{array}{l}
x_{1}(t) \\
x_{2}(t)
\end{array}\right]+\left[\begin{array}{cc}
0 & 0 \\
0.12 & 0
\end{array}\right] \times\left[\begin{array}{l}
x_{1}(t-0.05) \\
x_{2}(t-0.05)
\end{array}\right] \text {. }
$$

The zero solution of the system is exponentially mean square stable provided $\lambda(1-\alpha)>$ 1.2173 .

Proof Checking the eigenvalues of $A$, we find that they are 0.0121 and 0.9879 . By Theorem 1, we take the values are $\mu(A)=1.2029,\|B\|=0.12$ and $\|\Delta A\| \leq a=0,\|\Delta B\| \leq b=0$, using the matrix norm and the matrix measure. Then

$$
\begin{aligned}
& 2 a^{2}+2(b+\|B\|)^{2}+2 k<0, \\
& 2 a^{2}+2(b+\|B\|)^{2}+2(\mu(A)-\lambda(1-\alpha))<0, \\
& 2(0.12)^{2}+2(1.2029)-2 \lambda(1-\alpha)<0, \\
& \lambda(1-\alpha)>1.2173 .
\end{aligned}
$$

This system without impulses is unstable, but by Theorem 1 this system can be exponentially mean square stable.

\section{Acknowledgements}

This work was supported by the Science \& Engineering Research Board (DST-SERB) project file number: ECR/2015/000301 in India, the National Natural Science Foundation of China $(11301308,61673247)$, and the Research Fund for Distinguished Young Scholars and Excellent Young Scholars of Shandong Province (JQ201719, ZR2016JL024).

\section{Competing interests}

The authors declare that they have no competing interests.

\section{Authors' contributions}

All authors read and approved the final manuscript.

\section{Author details}

${ }^{1}$ Department of Mathematics, Amrita School of Engineering, Amrita Vishwa Vidyapeetham, Coimbatore, India. ${ }^{2}$ School of Mathematics and Statistics, Shandong Normal University, Ji'nan, P.R. China.

\section{Publisher's Note}

Springer Nature remains neutral with regard to jurisdictional claims in published maps and institutional affiliations.

Received: 1 July 2017 Accepted: 15 January 2018 Published online: 24 January 2018

\section{References}

1. Yang, Z, Xu, D: Robust stability of uncertain impulsive control systems with time varying delay. Comput. Math. Appl. $53,760-769(2007)$

2. Li, X, Wu, J: Stability of nonlinear differential systems with state-dependent delayed impulses. Automatica 64, 63-69 (2016) 
3. Niculescu, SI: Delay Effects on Stability: A Robust Approach. Springer, New York (2001)

4. Xu, DY: Robust stability analysis of uncertain neutral delay differential systems via difference inequality. Control Theory Adv. Technol. 5, 301-313 (1989)

5. Xu, DY: Robust stability of neutral delay differential systems. Automatica 30, 703-706 (1994)

6. Cao, DQ, He, P, Zhang, K: Exponential stability criteria of uncertain systems with multiple time delays. J. Math. Anal. Appl. 283, 362-374 (2003)

7. Liu, B, Dou, C, Hill, D: Robust exponential input-to-state stability of impulsive systems with an application in micro-grids. Syst. Control Lett. 65, 64-73 (2014)

8. Liu, B, Hill, D: Uniform stability and ISS of discrete-time impulsive hybrid systems. Nonlinear Anal. Hybrid Syst. 4, 319-333 (2010)

9. Kharitonov, VL, Zhabko, AP: Robust stability of time-delay systems. IEEE Trans. Autom. Control 3, 2388-2397 (1994)

10. Lu, J, Ho, DWC, Cao, J: A unified synchronization criterion for impulsive dynamical networks. Automatica 46, 1215-1221 (2010)

11. Yuan, L: Robust analysis and synthesis of linear time-delay systems with norm-bounded time-varying uncertainty Syst. Control Lett. 28, 281-289 (1996)

12. Samoilenko, AM, Perestyuk, NA: Impulsive Differential Equations. World Scientific, Singapore (1995)

13. Li, X, Cao, J: An impulsive delay inequality involving unbounded time-varying delay and applications. IEEE Trans. Autom. Control 62(7), 3618-3625 (2017)

14. Lakshmikantham, V, Bainov, DD, Simeonov, PS: Theory of Impulsive Differential Equations. World Scientific, Singapore (1989)

15. Li, X, Bohner, M, Wang, C: Impulsive differential equations: periodic solutions and applications. Automatica 52 173-178 (2015)

16. Li, X, Song, S: Stabilization of delay systems: delay-dependent impulsive control. IEEE Trans. Autom. Control 62(1), 406-411 (2017)

17. $L i, X$, Song, S: Impulsive control for existence, uniqueness and global stability of periodic solutions of recurrent neural networks with discrete and continuously distributed delays. IEEE Trans. Neural Netw. Learn. Syst. 24, 868-877 (2013)

18. Li, X, Zhang, X, Song, S: Effect of delayed impulses on input-to-state stability of nonlinear systems. Automatica 76, 378-382 (2017)

19. Zhang, X, Li, X: Input-to-state stability of non-linear systems with distributed-delayed impulses. IET Control Theory Appl. 11(1), 81-89 (2017)

20. Stamova, I, Stamov, T, Li, X: Global exponential stability of a class of impulsive cellular neural networks with supremums. Int. J. Adapt. Control Signal Process. 28, 1227-1239 (2014)

21. Wu, SJ, Guo, XL, Lin, SQ: Existence and uniqueness of solutions to random impulsive differential systems. Acta Math. Appl. Sin. 22(4), 595-600 (2006)

22. Anguraj, A, Vinodkumar, A: Existence, uniqueness and stability results of random impulsive semilinear differential systems. Nonlinear Anal. Hybrid Syst. 4, 475-483 (2010)

23. Agarwal, R, Hristova, S, O'Regan, D: Exponential stability for differential equations with random impulses at random times. Adv. Differ. Equ. 2013, 372 (2013)

24. Wu, SJ, Meng, XZ: Boundedness of nonlinear differential systems with impulsive effect on random moments. Acta Math. Appl. Sin. 20(1), 147-154 (2004)

25. Wu, SJ, Duan, YR: Oscillation, stability, and boundedness of second-order differential systems with random impulses. Comput. Math. Appl. 49(9-10), 1375-1386 (2005)

26. Wu, SJ, Guo, XL, Zhou, Y: $p$-Moment stability of functional differential equations with random impulses. Comput. Math. Appl. 52, 1683-1694 (2006)

27. Anguraj, A, Wu, S, Vinodkumar, A: Existence and exponential stability of semilinear functional differential equations with random impulses under non-uniqueness. Nonlinear Anal. TMA 74, 331-342 (2011)

28. Anguraj, A, Vinodkumar, A, Malar, K: Existence and stability results for random impulsive fractional pantograph equations. Filomat 30(14), 3839-3854 (2016)

29. Vinodkumar, A, Malar, K, Gowrisankar, M, Mohankumar, P: Existence, uniqueness and stability of random impulsive fractional differential equations. Acta Math. Sci. Ser. B 36(2), 428-442 (2016)

30. Vijay, S, Loganathan, C, Vinodkumar, A: Approximate controllability of random impulsive semilinear control systems. Nonlinear Stud. 23(2), 273-280 (2016)

31. Liu, B, Hill, DJ, Sun, ZJ: Mixed $\mathscr{K}$-dissipativity and stabilization to ISS for impulsive hybrid systems. IEEE Trans. Circuits Syst. Il, Express Briefs 62, 791-795 (2015)

\section{Submit your manuscript to a SpringerOpen ${ }^{\mathcal{O}}$ journal and benefit from:}

- Convenient online submission

- Rigorous peer review

- Open access: articles freely available online

- High visibility within the field

- Retaining the copyright to your article

Submit your next manuscript at $\boldsymbol{\triangleright}$ springeropen.com 